\title{
Pensar las escalas para pensar las luchas
}

\author{
Think about the scales to think about the struggles
}

\section{Pense sobre as escalas para pensar sobre as lutas}

\author{
Mathieu UHEL* \\ Traducción Luz Vanessa Pérez Tapia \\ Doctora en Geografía, UNAM \\ Docente Universidad Anáhuac México, México. \\ Docente invitada de la Maestría en Ordenamiento Territorial y Planificación \\ Urbana, Universidad Mayor de San Andrés, Bolivia \\ luzvan perez@comunidad.unam.mx
}

UHEL, Mathieu (2019). "Penser les echelles pour penser les luttes", en Brault A. y S. Le Roulley (eds). Croniques du Désert. Le désert urbain.Caen-Francia, Éditions Grévis. Pp. 167-186

\begin{abstract}
RESUMEN
A través de un título sugerente, "pensar las escalas para pensar las luchas", Mathieu Uhel entreteje la construcción teórico-crítica del concepto escala, generada por la geografía radical anglosajona de finales del siglo XX, con la necesidad/utilidad práctica de la escala para concienciar las luchas sociales. El artículo cumple un doble propósito: por un lado, delinear los elementos de lectura sobre el concepto escala; $y$, con ello, promover la atención de esta problemática en las luchas contemporáneas. En un primer apartado, Uhel ubica las discusiones académicas en torno a la escala, como herramienta metodológica útil para comprender la complejidad de las sociedades capitalistas; en el segundo apartado, el autor avanza la exposición en torno al contexto de la dimensión escalar del imperialismo capitalista; finalmente, el autor se centra en el rol de la actividad política a escala nacional en la tensa relación entre las imposiciones del capital y la lucha social.
\end{abstract}

\begin{abstract}
Mathieu Uhel's suggestive title, "Thinking about scales to think about struggles", he interweaves the theoretical-critical construction of concept scale, generated by radical Anglo-Saxon geography in the late 20th century, with it's practical utility to social struggles. The article serves two purposes: on the one hand, Uhel locates academic discussion around scale; and, with this, he promotes attention to this problem in contemporary struggles. In the first section, Uhel locates academic discussions around scale, as a useful methodological tool to understand the complexity of capitalist societies; in the second section, the author advances the argument around the context of the scalar dimension of capitalist imperialism; finally, the author focuses on the role of political activity on a national scale in the tense relationship between the impositions of capital and the social movement.
\end{abstract}

*Sobre el autor

Mathieu Uhel es geógrafo, profesor del Instituto Universitario de Tecnología en Alençon y miembro del equipo del Laboratorio Espacios y Sociedades de la Universidad de Caen Normandía, Francia.

\section{Citar como:}

Pérez, L. (2021). Pensar las escalas para pensar las luchas. Espiral, revista de geografías y ciencias sociales, 3(5), 135-146. https:// dx.doi.org/10.15381/espiral.v3i5.21182 


\section{RESUMO}

Por meio de um título sugestivo, "pensando escalas para pensar lutas", Mathieu Uhel entrelaça a construção teórico-crítica do conceito de escala, gerado pela geografia radical anglo-saxônica do final do século XX, com a necessidade / utilidade prática da escala para aumentar a consciência das lutas sociais. $\mathrm{O}$ artigo tem um duplo propósito: por um lado, delinear os elementos de leitura sobre o conceito de escala; e, com isso, promover atenção a esse problema nas lutas contemporâneas. Na primeira seção, Uhel localiza as discussões acadêmicas em torno da escala, como uma ferramenta metodológica útil para compreender a complexidade das sociedades capitalistas; na segunda seção, o autor avança a exposição em torno do contexto da dimensão escalar do imperialismo capitalista; por fim, o autor enfoca o papel da atividade política em escala nacional na tensa relação entre as imposições do capital e a luta social.

PALABRAS CLAVE: escalas; geografía radical; acumulación capitalista.

KEYWORDS: scales; radical geography; capitalist accumulation.

PALAVRAS CHAVES: escalas; geografia radical; acumulação capitalista.

\section{Comentario a la edición en español}

"La geografía puede servir a nuestras luchas" nos dice enfático Mathieu Uhel. Ello nos recuerda de inmediato, aunque en sentido inverso, al libro "La geografía: un arma para la guerra" (1976) del también geógrafo francés Ives Lacoste. Entre muchas otras cosas, Lacoste señala el poder que implica el conocimiento geográfico; por su parte, Uhel reivindica el uso del saber geográfico más allá de su instrumentalización para la dominación, de hecho señala su valor para la organización política, entre pares, a distintas y distantes escalas geográficas; como posibilidad "de organizar la producción y la reproducción de la vida cotidiana y de resistir a la opresión y a la explotación a una escala superior" -nos dijo un día el buen geógrafo escocés Neil Smith (1992: 60). En este sentido, el uso de la escala -mejor dicho- la organización social con perspectiva escalar, como sugiere Uhel, no sólo implica el reconocimiento del territorio inmediato y de los impactos de los proyectos políticos estatales y de los grandes capitales que inciden en las formas de reproducción de la vida cotidiana, también permite vislumbrar la articulación territorial de las luchas sociales con otros y múltiples proyectos afines.

Y es que más allá de su dimensión geométrica (Silveira, 2015) la escala constituye una dimensión de lo social. De acuerdo con González (2005) la idea de escala sale a la luz en medio de las discusiones geográfico-sociológicas anglosajonas en torno a la conceptualización de la "localidad", ello como resonancia del proceso de globalización, definida por Santos como "la introducción en el sistema-mundo de todos los lugares y de todos los individuos aunque en diversos grados" (1993: 69). Entre líneas, en la exposición de Santos se aprecia la realidad e importancia de la escala que, al igual que el espacio, es resultado de la producción socialmente determinada; no obstante "la producción de la escala puede ser la diferenciación más elemental del espacio geográfico y es en toda su extensión un proceso social" (Smith, 2015: 141).

Es así que la escala puede ser vista como una propiedad del objeto (geográfico) tanto como un elemento metodológico para la investigación (y para la acción -quizá añadiría Uhel). Por ejemplo, en la búsqueda de su aplicación para el estudio de problemas socio-ambientales, Rivera y Galicia (2016: 140) refieren tres componentes para la construcción metodológica de la perspectiva escalar: la magnitud de los fenómenos (extensión, resolución y nivel); la identificación de propiedades específicas (jerarquía y dependencia escalar); y la unidad del área modificable (definición de alcances de las interferencias como consecuencia de la agregación de los datos).

Además de su utilidad metodológica, la escala es una propiedad de lo social: "las sociedades también producen escala" nos recuerda Smith (Op. Cit.). Y ésta es quizá la 
mayor motivación de Uhel: ¿cómo producir y/o utilizar a conciencia la escala para la organización del movimiento social?

En suma, desde una óptica especialmente afín a Neil Smith, Uhel concluye su reflexión al subrayar la utilidad de la escala para pensar táctica y estratégicamente las respuestas locales, las luchas sociales, pero también -y quizá en el mismo sentidopara remover los afectos y revitalizar las sensibilidades solidarias, no sólo en el calor de las centralidades y de los acontecimientos sino incluso en la escala de la crítica a la vida cotidiana.

\section{Pensar las escalas para pensar las luchas}

Las luchas políticas o sindicales se afirman en múltiples escalas. Desde el movimiento social tipo "movimiento contra la ley del trabajo y su mundo" hasta una huelga localizada, las estrategias y las tácticas son dependientes tanto de su inscripción en un "movimiento de masas", como de su inscripción en una escala. Pensar las escalas aparece entonces primordial para orientar las decisiones estratégicas.

Frecuentemente, durante periodos en conflicto los "sindicalistas combativos", aquellos en "ruptura" con su organización o al margen, incluso los autónomos intentan resonar con las luchas localizadas, a fin de estar a la altura de sus experiencias. Aunque este "pensamiento escalar" se afirme tácitamente, no necesariamente es considerado a la altura de las potencialidades tácticas y estratégicas que genera ni del movimiento que supone.

Lejos de bosquejar aquí las pistas propiamente políticas, propongo dar los elementos de lectura sobre el concepto de escala, menos trabajado en Francia que en los países anglofónos. Para esta síntesis, la idea es promover la atención de esta problemática en las luchas contemporáneas, en los análisis de las relaciones de poder e igualmente en la elaboración de las prácticas defensivas y ofensivas. Una vez presentado el lugar que ocupa el concepto dentro de la geografía anglosajona y aportados los elementos de definición de las escalas ligados al concepto de poder, buscaré inscribir estos desarrollos dentro del contexto de las sociedades capitalistas contemporáneas, ello con la ayuda de trabajos de geógrafos radicales. Si "la geografía sirve para hacer la guerra" estemos seguros que ella también puede servir a nuestras luchas.

\section{El concepto de escala en la geografía anglófona}

A partide los años 1980, la geografía radical anglófona ha multiplicado los trabajos sobre las escalas (scalar turn) como prolongación del materialismo histórico-geográfico de David Harvey (Keil y Mahon, 2009). Como ejemplo, es posible distinguir a sus precursores: Peter Taylor (1982), Neil Smith (1992; 1993) o inlcuso David Delaney y Helga Leitner (1997). Las raíces económico políticas de sus investigaciones, desarrolladas en la geografía radical, puede explicar que los análisis hayan privilegiado los objetos tales como el capitalismo y el Estado, focalizando una escala particular, la escala nacional, en detrimento de las relaciones entre escalas y sus procesos de construcción, deconstrucción, reconstrucción y oposición. Entre los precursores y las investigaciones contemporáneas, los geógrafos críticos han buscado complejizar la teorización de la dimensión escalar de las sociedades hasta llevar su mirada sobre otros objetos de investigación como el género, la "raza", el ambiente e incluso los movimientos sociales. Si bien algunos geógrafos han elaborado una hibridación del concepto de escala con el de red (Swyngedou et Brenner), otros han intentado amironar el recurso a las escalas poniendo el acento sobre las redes (Cox y Massey), mientras que ciertos geógrafos han promovido un abandono puro y simple del concepto de escala (Allen y Marston). 
En la obra Lost geographies of power, John Allen reafirma la importancia de la dimensión espacial en el ejercicio de poder. El poder es un efecto relacional de las interacciones sociales, a diferencia de los recursos atrapados en una tensión entre fijación y movimiento. De acuerdo con éste geógrafo, el ejercicio de poder "compensa la distancia entre aquí y allá" entre el lugar de movilización de los recursos y el lugar de producción de sus efectos; "a través de la sucesión de relaciones intermediarias o a través del establecimiento de una presencia simultánea" (Allen, 2003: 2). En defensa de una "visión topológica de las relaciones sociales", John Allen rechaza el concepto de escala en provecho del concepto de red. En la misma línea, Sallie Marston, Jean-Paul Jones III y Keith Woodward critican la supuesta jerarquía intrínseca de las escalas. Ellos defienden una ontología plana, en apoyo de trabajos de Bruno Latour o de Gilles Deleuze y Félix Guattari, que privilegia una aproximación horizontal de la sociedad donde los sitios están relacionados por las redes (Marston, et al., 2005). En respuesta a esta ofensiva contra las escalas, Helga Leitner y Byron Miller reconocen la inmensa importancia convenida a las escalas dentro de la literatura geográfica y a la concepción jerarquizada procedente de las escalas. No obstante, ellos refutan el llamado de los autores a suprimir las escalas y a recurrir a una ontología plana (Leitner y Miller, 2007), consideran que Marston et al. parten de presupuestos erróneos sobre las escalas y movilizan un marco teórico idealista del actor-red.

De esta forma, Marston et al. confunden jerarquía y verticalidad en la definición de las escalas e ignoran las jerarquías de poderes en la reproducción de desigualdades en las redes. Helga Leitner y Byron Miller defienden a la inversa que:

"el reconocimiento de los arreglos escalares y de la existencia de asimetrías del poder es crucial para una política progresista, a la vez en términos de desarrollo de espacios de alternativa política y el despliegue de estrategias socio-espaciales de resistencia" (Leitner y Miller, 2007: 9)

Este debate sobre el concepto de escala dentro de la geografía anglófona ha permitido clarificar la posición de los geográfos así como complejizar el análisis de la dimensión escalar de las sociedades capitalistas.

De este modo, podemos decir que los geógrafos radicales se oponen a una definición de las escalas como espacios delimitados, jerarquizados, ajustados e inflexibles. Ellos comprenden el concepto como una construcción social, procesual y relacional, ligada a las relaciones sociales de poder y por lo tanto a la política (Brenner, 2001). De esta forma, las escalas tienen una existencia social, material e ideal (Swyngedouw, 1997) o, para retomar la definición de Frabrice Ripoll, son “a la vez producto objetivo de las prácticas y relaciones sociales y representación jerarquizada de la realidad social" (2005). Por su parte, el geógrafo Erik Swyngedouw y el sociólogo Neil Brenner han aportado, en los últimos años, importantes contribuciones al análisis de la dimensión escalar de las sociedades. Ellos definen las escalas con relación a la evolución de las relaciones sociales de poder.

Para Swyngedow:

"cambiando la importancia y el rol de ciertas escalas geográficas, reafirmando la importancia de otras escalas, creando algunas veces nuevas escalas significativas, pero -lo más importante- esta redefinicón de las escalas altera y manifiesta las variaciones de la geometría del poder social reforzando el poder y el control de algunos y reduciendo el poder de otros" (Swyngedouw, 2004: 19)

Al hablar del proceso de estructuración escalar, Neil Brenner añade igualmente que la organización de jerarquías escalares conlleva la exclusión y la inclusión, la dominación y la subordinación de algunos actores, alianzas y organizaciones en detrimento de 
otros, siguiendo los criterios de clase, de género, de "raza" y de nacionalidad. También subraya que las jerarquías escalares no son solamente las arenas donde se desarrollan las luchas de poder, ellas son el objeto mismo de las luchas por el poder.

El resultado del proceso de estructuración escalar da lugar a aquello que Neil Brenner nombra scalar fixes (fijaciones, arreglos o disposiciones escalares), que constituyen jerarquías geográficas relativamente fijas en las cuales las actividades políticas, económicas y culturales organizadas a deteminadas escalas tienden a predominar sobre otras escalas (Ibid.). Podríamos entonces hablar de escala(s) de referencia o dominante(s) al interior de un arreglo escalar. Las jerarquías escalares provenientes de los procesos de estructuración deben ser entendidas no como una pirámide de escalas ajustadas y encerradas, sino como un mosaico de geometrías escalares desigualmente superpuestas y densamente interestratificadas. En este sentido, en la búsqueda de articular los conceptos de escala y de red, Neil Brenner afirma que las escalas evolucionan relacionalmente en jerarquías enmarañadas y en redes escalares dispersas.

A inicios de los años 1990, a propósito de una experiencia político-artística cercana a las personas sin techo de la ciudad de Nueva York, Neil Smith acuñó la expresión "políticas de escala". Al respecto el geógrafo afirma que los grupos sociales tienen:

“la posibilidad de saltar las escalas -de organizar la producción y la reproducción de la vida cotidiana y de resistir a la opresión y a la explotación a una escala superior- hacia un campo geográfico más amplio"(Smith, 1992: 60)

Desde hace tiempo, la política escalar ha estado asociada a los movimientos sociales y a una visión de la acción colectiva ascendente ("salto escalar") $)^{1}$. Con voluntad de ampliar y complejizar esta definición, Neil Brenner, introduce una distinción entre políticas de escala en plural y en singular. En singular (la política de escala), es una mezcla de "la producción, la reconfiguración o la contestación de un cierto aspecto de la organización socio-espacial al interior de una arena geográfica relativamente delimitada", mientras que en plural (la política de las escalas) es una mezcla de "la producción, la reconfiguración o la contestación de las diferenciaciones, ordenamientos y jerarquías particulares entre las escalas geográficas" (Brenner, 2001: 599-600). Para el sociólogo, esta última acepción permite no solamente incluir la producción, la reconfiguración y la contestación de una escala particular sino también las relaciones de las escalas entre ellas. Más que de una o de las políticas de escala, Neil Brenner propone hablar de políticas de estructuración escalar o más simplemente de politics of scaling. Esta última expresión difícil de traducir en francés podría ser reemplazada por la expresión "política multiescalar", como lo sugiere la socióloga Dominique Masson (2009).

Si bien la estructuración escalar no puede ser reducida a los procesos económicopolíticos, me parece pertinente sin embargo tomar en cuenta las lógicas del capital y del Estado, asociadas a las relaciones sociales de clase que ellos inducen, con el fin de comprender la evolución de la organización de las escalas en las sociedades capitalistas.

\section{La dimensión escalar del imperialismo capitalista}

En el contexto capitalista, la relación social de producción está fundada sobre la propiedad privada de los medios de producción y la explotación de la fuerza de trabajo a partir de la cual aquellos que detentan el capital obtienen un plusvalor, 1 Esta concepción centrada sobre la acción ascendente del movimiento social se aproxima, en mi opinión, a la definición del cambio de escala desarrollado por el politólogo Sidney Tarrow y los sociólogos Doug Mc Adam y Charles Tilly. Ver Sidney G Tarrow y Doug McAdam, "Scale shift in transnational contention”, en Transnational protest and global activism. People, passions and power, Lanham, Rowman \& Littlefield Publishers, 2005, pp. 121-147; ver igualmente Charles Tilly y Sidney G Tarrow, Politique(s) du conflit: de la grève à la rèvolution, Paris, Presses de Sciences Po, 2015. 
su fuente de ganancia. Esta búsqueda de ganancia es el motor del capitalismo, aquello que los economistas han llamado el proceso de acumulación de capital. Se trata de reinvertir una parte del plusvalor retirado de la explotación de la fuerza de trabajo a fin de engrandecer sin cesar las capacidades de producción en vista de retirar siempre más ganancia. La dimensión espacial de proceso de acumulación de capital es tomado como una contradicción fundamental, entre fijación y movimiento, contradicción que David Harvey nombra lógica molecular de acumulación de capital (Harvey, 2005). Efectivamente, retomando a Neil Brenner, la acumulación de capital se esfuerza por "aniquilar el espacio por el tiempo" (Marx), esto significa abatir las barreras geográficas que frenan la circulación del capital y por tanto la realización de la ganancia. Pero para perseguir esta dinámica continuada de desterritorialización y de compresión espacio-temporal (Harvey), "el capital necesita infraestructuras territoriales relativamente fijas e inmóviles (...) inscritas a escalas geográficas múltiples y entrelazadas" (Brenner, 1998). De esta forma, la territorialización del capital se realiza a través de la producción de arreglos escalares, que delimitan y jerarquizan las relaciones sociales capitalistas. Puesto que el proceso de acumulación de capital no puede ser tomado independientemente de las relaciones sociales de clase, estos arreglos escalares son el objeto de rechazo, de proyectos alternativos y de conflictos, sometidos a la lucha de clases (Gough, 2004). Las clases y las fracciones de clase desarollan una política escalar que produce, defiende o contesta la estructuración escalar existente de las relaciones de poder en función de sus intereses.

El proceso de acumulación de capital siendo contradictorio y conflictual, del que las crisis sobrevienen regularmente, necesita de los mecanismos y de las instituciones para superarlas y estabilizar la máquina de la ganancia. En esta perspectiva, el Estado inteviene para garantizar y facilitar la dinámica global del capitalismo, regular los conflictos sociales y arbitrar los conflictos de interés entre las diferentes fracciones del capital (Harvey, 1985). David Harvey propone que la lógica estatal es diferente de la lógica molecular de acumulación de capital, porque éste evoluciona en un espacio segmentado en territorios y, en las democracias, en un tiempo dictado por los ciclos electorales (Harvey, 2005). Por su parte, Neil Brenner subraya que el Estado juega un rol central en la producción de arreglos escalares por el proceso de acumulación de capital, facilitando la territorialización de las relaciones sociales capitalistas. Sin embargo, ello no significa que el Estado intervenga sistemáticamente en favor del capital en detrimento del trabajo. Las contradicciones entre clases y fracciones de clases repercuten al interior del Estado. Así mismo, el sociólogo propone que la estructura escalar del Estado puede ser modificada en función de las crisis del capitalismo (Brenner, 1998).

Las lógicas capitalistas y estatales se combinan de manera compleja y contradictoria para engendrar aquello que David Harvey llama el imperialismo capitalista:

"la fusión contradictoria de la política estatal-imperial (el imperialismo como proyecto político específico de agentes, cuyo poder está fundado sobre el control de un territorio y la capacidad de movilizar sus recursos humanos y naturales con propósitos políticos, económicos y militares) con los procesos moleculares de acumulación de capital en el espacio y en el tiempo (el imperialismo como proceso políticoeconómico difuso, en el cual el punto primordial es el control y el uso del capital)" (Harvey, 2005: 26)

La combinación de estas lógicas participan de la estructuración escalar del sistema mundo capitalista. De acuerdo con David Harvey, la evolución de la geografía del imperialismo capitalista, y por tanto su dimensión escalar, deriva de las soluciones espacio-temporales encontradas por los poseedores del capital de cara a las crisis recurrentes y sistemáticas del capitalismo. 
En su aproximación geográfica del imperialismo capitalista, David Harvey reevalúa y "espacializa" la teoría general de la acumulación de capital de Marx. Esto proviene de "la acumulación fundada sobre la depredación, la estafa y la violencia en una 'etapa original' considerada como anticuada o, de acuerdo con Rosa Luxemburgo, como de una cierta manera 'exterior' al sistema capitalista" (Ibid.). A la inversa, Harvey sostiene que las prácticas predatorias de la acumulación "primitiva" u "originaria" persisten durante toda "la larga geografía histórica de la acumulación del capital". Así mismo, reintroduciendo el espacio en el materialismo histórico del filósofo aleman, el geógrafo radical anglófono muestra que el espacio (tal como el tiempo) es crucial en la superación de las crisis del capitalismo y la reactivación del proceso de acumulación de capital.

Estas crisis periódicas y recurrentes de la acumulación de capital se manifiestan por el exceso de capitales (mercantiles, dinero o capacidad de producción) y de fuerza de trabajo. Para frenar la caída tendencial de la tasa de ganancia y evitar la devaluación de los activos, hay que encontrar las formas rentables que permitan absorber los excedentes de capital. David Harvey propone que estas crisis de sobreacumulación podrían ser superadas por aquello que él llama las soluciones espacio-temporales:

"la expansión geográfica y la reorganización espacial ofrecen esta posibilidad [de absorción de los excedentes], pero ellas no podrían estar separadas por retrasos temporales por los cuales el exceso de capital es invertido en proyectos a largo plazo" (Ibid.: 65)

Entre otras soluciones espacio-temporales, se puede invertir en la construcción de infraestructuras o en gastos sociales (educación o investigación), en una nueva organización de la división del trabajo, en el acceso a los recursos inéditos y menos caros, en la promoción de nuevas regiones como espacios dinámicos de acumulación de capital, en la penetración de formaciones sociales preexistentes a las relaciones sociales y a los dispositivos institucionales capitalistas. Estas estrategias entrañan expansiones, reorganizaciones y reestructuraciones geográficas que pueden tomar la forma de mecanismos violentos y destructores. Los reordenamientos escalares constituyen una de las manifestaciones de estas soluciones espacio-temporales a las crisis del captialismo.

David Harvey subraya que la investigación de las soluciones espacio-temporales es un proceso contradictorio, porque los nuevos espacios dinámicos de acumulación de capital generan excedentes y buscan las soluciones para absorberlos a través de sus propias expansiones geográficas. Esta contradicción desemboca en el desarrollo de crisis itinerantes o a la exacerbación de la competencia internacional.

\section{La geografía histórica del imperialismo capitalista: arreglos escalares y escala de referencia.}

La geografía histórica del imparialismo capitalista de David Harvey (2005) pone en evidencia tres etapas: el ascenso de los imperialismos burgueses europeos (18811945), el "super-imperialismo" estadounidense (1945-1970), el neoliberalismo o nuevo imperialismo (a partir de los años 1970). Neil Brenner (1998) distingue tres periodos, que corresponden a la periodización de David Harvey, en los cuales el Estado es intervenido de una manera determinante en la producción de arreglos escalares por el proceso de acumulación de capital seguido de crisis mayores: el aislamiento al interior de la escala nacional de la organización territorial capitalista (1890-1930); el reforzamiento de la escala nacional en el ciclo keynesiano-fordista de crecimiento capitalista (1950-1970); la desnacionalización de la organización territorial capitalista y la búsqueda de arreglos escalares "glocales" alternativos (a partir de los años 1970). De modo esquemático, en los próximos párrafos sólo mencionaré las dos fases principales que consisten en la construcción y la deconstrucción de la escala nacional 
como escala dominante al interior del arreglo escalar de las relaciones de poder económico-político contemporáneas.

La construcción de un arreglo escalar, en el cual la escala nacional es dominante, se remonta a la segunda mitad del siglo XIX, época que David Harvey considera como ascenso de los imperialismos burgueses. Durante este periodo, situado bajo la hegemonía internacional de la Gran Bretaña, el capital y el Estado de los países del centro se asociaron para aumentar y extender la explotación de las periferias del sistema mundo capitalista (Harvey, 2005). Por otra parte, Neil Brenner (1998) afirma que asistimos a un confinamiento de las relaciones sociales en el territorio nacional del Estado y a una convergencia estructural creciente entre las escalas de la organización industrial capitalista y las escalas de las instituciones estatales. El aparato de estado profundiza su control sobre el territorio nacional intentando integrar las escalas infranacionales e interviniendo de manerea creciente en los conflictos sociales entre capital y trabajo. Esta primer fase finalizó con la crisis económica de los años 1930 y la intensificación de las rivalidades geopolíticas interimperialistas, y desembocó en dos guerras mundiales.

Después de la Segunda Guerra Mundial, las grandes potencias capitalistas, bajo la dirección de Estados Unidos, buscaron establecer

"un contrato (...) para evitar toda guerra intestina y encontrar un medio racional de abordar colectivamente la cuestión de la sobreacumulación, que estuvo en el origen del desastre de los años 1930" (Harvey, 2005)

Se trataba de repartir las ventajas/beneficios del desarrollo de un capitalismo integrado en los centros y de comenzar una nueva forma de expansión geográfica en las periferias. Los excedentes de capital estadounidense fueron invertidos en la reconstrucción europea y las perspectivas de beneficio ligadas al proceso de descolonización empujaron a Estados Unidos a estimular la independencia de los países con respecto a la vieja Europa. En esta segunda fase del imperialismo capitalista, Estados Unidos ocupó una posición de superpoderío, más política y militar que económica, notoriamente gracias a la rivalidad mundial con Rusia durante la "guerra fría". David Harvey afirma que

"la acumulación por desposesión se volvió relativamente sutil durante este periodo, lo que predominó fue el resultado de las luchas (de clases) en los años 60 y 70 respecto de la reproducción ampliada del capital" (Ibid.)

El desarrollo de un capitalismo integrado en las regiones del centro se institucionaliza en aquello que acostumbramos llamar el acuerdo fordista-keynesiano. Los gastos públicos y la intervención directa del Estado en la economía responde a las necesidades del régimen de acumulación y se amoldan parcialmente a las reivindicaciones sociales. Durante este periodo de consolidación de la escala nacional, el Estado deviene esencial

"en la promoción, la regulación y el financiamiento del desarrollo industrial capitalista -sobre todo a través [de su] rol en la construcción de infraestructuras territoriales a gran escala para la producción industrial, el consumo colectivo, el transporte y la comunicación" (Brenner, 1999: 44-45)

De frente al desarrollo desigual del territorio, el estado ha intentado reducir las desigualdades entre ciudades y regiones a través del establecimiento de políticas redistributivas (Brenner, 1998). La escala nacional ha devenido la escala de referencia del desarrollo económico y de la contestación sociopolítica. La política escalar del movimiento obrero ha consistido en exceder la escala local del taller o la fábrica y ha aumentado la influencia económico-política de las organizaciones sindicales a la 
escala nacional, operando de este modo en un salto escalar (Swyngedouw, 2004). Los intereses de clase son regulados por las negociaciones entre representates del capital y del trabajo a la escala nacional por intermediación del Estado (Uitermark, 2002). Más precisamente todavía,

"las luchas políticas entre diferentes facciones de clase -a todas las escalas pero sobre todo a la escala del Estado-Nación- son (parcial y temporalmente) pacificadas a través de la incorporación de fracciones del trabajo al interior de las instituciones del Estado" (Neocleous, 1996: 248. Citado en Uitermark, 2002)

El movimiento obrero se encuentra controlado e instrumentalizado por el Estado a la escala nacional en favor de la reproducción de la relaciones sociales capitalistas.

Con la crisis económica de los años 1970, asistimos a un proceso de desnacionalización de la escala dominante, del arreglo escalar del acuerdo fordista-keynesiano, y al desarrollo de un proceso de "glocalización" como lo define Erik Swyngedow. Se trata de un doble proceso que se caracteriza por el despliegue de estrategias de localización global de las diferentes fracciones del capital y, por otro lado, por la reestructuración del Estado a la escala nacional hacia la alta (escalas supranacionales) y hacia la baja (escalas infranacionales) (Swyngedow, 1997; 2004).

Las clases dominantes capitalistas de los países del centro emprenden una ofensiva ideológica y política con el fin de detener la caída de la tasa de ganancia, así como relanzar el proceso de acumulación de capital. La acumulación por desposesión, que sigue este mismo camino, no se contenta solamente con los recursos de los mecanismos tradicionales tales como la privatización y la mercantilización de las tierras en los países de la periferia o la apropiación del "salario social" heredada de los "treinta gloriosos" en los países del centro. Ella parte a la conquista de todos los sectores o bienes exluidos de la valorización mercantil capitalista hasta el presente, tales como la genética, la cultura o los bienes ecológicos planetarios; la mercantilización y la privatización de los bienes y servicios públicos; la biopiratería; la extensión de los derechos de propiedad intelectual; la mercantilización y privatización de la cultura, de las historias y de la creatividad intelectual (Harvey, 2005). La expansión de las firmas transnacionales y del capital financiero, asociado al rol de las Nuevas Tecnologías de la Información y de la Comunicación (NTIC) en las transacciones económicas, aceleran la circulación del capital en la economía mundial (Brenner, 1998). Con todo, conforme a la lógica molecular de la acumulación del capital, estas inversiones necesitan un anclaje territorial. La escala nacional de las negociaciones entre capital y trabajo y de intervención del Estado es cada vez más eludida por los propietarios del capital en beneficio de las escalas urbana y regional.

Bajo la motivación de los Estados Unidos y de la Gran Bretaña, las medidas neoliberales, promueven la desregulación, la liberalización y la privatización de las economías nacionales, se mundializan. Esta mundialización es, por otra parte, facilitada por el fin de la "guerra fría" y la caída del bloque soviético y se va a imponer en los países de la periferia. Las Instituciones Financieras Internacionales (IFI) y la Organización Mundial de Comercio (OMC) ven su función de origen reguladora orientarse hacia una misión tendiente a la apertura de los mercados y la circulación sin obstáculos para las mercancías y los capitales. El fomento al endeudamiento y en consecuencia el incremento de las tasas de interés, orquestados por las IFI y beneficiando exclusivamente a los Estados y bancas del centro, devienen priedra angular de la acumulación por desposesión en la periferia. La ortodoxia neoliberal es aplicada vía la adopción de Programas de Ajuste Estructural, con el objetivo proclamado de reducir el tamaño del Estado, desacreditado por la corrupción y la ineficiencia de la burocracia, y de sanear las finanzas públicas para la obtención de nuevos préstamos, 
condiciones supuestamente necesarias para el reestablecimiento del crecimiento económico.

En reacción a estas nuevas estrategias económicas "glocales", el Estado redefine su rol y su organización. La nueva política y la nueva estructuración escalar del Estado son respuestas defensivas frente a la intensificación de la competencia económica global, pero además éstas también constituyen una estrategia coordinada tendiente a facilitar y a coordinar el proceso de globalización del capital a todas las escalas. Neil Brenner y Erik Swyngedow precisan que el Estado ha promovido la globalización económica a escalas supra e infranacionales al mismo tiempo, con lo que ha transferido substanciales aspectos de sus competencias políticas a estas mismas escalas (Ibid.). La constitución de bloques económicos y políticos a la escala internacional, tales como ALENA [acrónimo en francés para Tratado de Libre Comercio de América del Norte, TLCAN] o la UE [Unión Europea], y la descentralización político-administrativa a escalas regionales, urbanas y locales, ilustran la política escalar del Estado en favor de procesos de acumulación de capital y la reestructuración escalar del aparato de Estado. En oposición de la política redistributiva fordista-keynesiana, el Estado ha comenzado a fomentar un alto nivel de desarrollo geográfico desigual, con el fin de que las ciudades y las regiones participen en una competencia intensificada por las inversiones del capital y las subvenciones públicas (Ibid.).

De cara a esta reestructuración "glocal" económico-política, las Organizaciones del Movimiento social (OMS) son desestabilizadas, en primera línea las organizaciones sindicales. Las estrategias de localización global del capital apuntan también a romper el poder de los sindicatos, adquirido a la escala nacional, y a relocalizar las negociaciones salariales a las escalas infranacionales. Las prácticas sindicales salidas del compromiso fordista-keynesiano, centradas sobre la escala nacional y la mediación estatal en el conflicto del trabajo, ya no están adaptadas a éste nuevo contexto. Las OMS deben repensar su modo de organización, su marco de interpretación y sus reivindicaciones, así como su repertorio de acción colectiva, esto es la definición y la ejecución de su política escalar.

\section{Conclusión}

Las consecuencias de la primavera de 2016 han estado marcadas por iniciativas que intentan articular las escalas en el periodo de recesión. Desde el llamado "nacional" ¡lo bloqueamos todo! a la creación de comités locales en torno a la dinámica del Frente Social, se han ensayado nuevas composiciones a partir de las prácticas comunes más que de los discursos o las propuestas ideológicas. Ciertamente, los intentos de recuperación han debilitado estas iniciativas cuando el carácter común de las fuerzas compuestas fue, por parte de algunos partidos en particular, asimilado a acciones unitarias más que a una articulación de prácticas específicas dirigidas a objetivos comunes. Alrededor de la afirmación de una práctica (el bloqueo de flujos), se constata una diversidad de otros modos de acción que cubren el amplio espectro de las prácticas contestatarias - de la petición al confrontamiento, de la marcha a la destrucción- se manifiestan de manera a la vez diferenciadas en función de las configuraciones locales, pero juntas resonando a la escala del territorio nacional.

En otros lugares, se están llevando a cabo otras iniciativas de composición congregando personas de diferentes culturas políticas o sindicales, incluso muy lejanas. Si la crítica de las burocracias sindicales ha podido ser asumida de manera bastante clásica en los ambientes libertarios, lejos de la efervescencia parisina (de la cabeza de la procesión), los encuentros hemos roto los esquemas perceptivos y reconfigurado las relaciones de camaradería, incluso, algunas veces, de amistad. Los afectos, también, remueven las escalas de la crítica. 


\section{Referencias Bibliográficas}

\section{Referencias de la edición en francés}

Allen, J. (2003). Lost geographies of power. Oxford: Blackwell publ.

Brenner, N. (1998). Between Fixity and Motion: Accumulation, Territorial Organization and Historical Geography of Spatial Scales. Environment and Planning D: Society and Space, 1 agosto 1998, vol. 16, no. 4, p. 461.

Brenner, N. (1999). Beyond State-Centrism? Space, Territoriality, and Geographical Scale in Globalization Studies. Theory and Society, vol. 28, no. 1, pp. 44-45.

Brenner, N. (2001). The limits to scale? Methodological, reflections on scalar structuration. Progress in Human Geography, 1 diciembre 2001, vol. 25, no. 4, pp. 591-614

Delaney, D. y Leitner, H. (1997). The political construction of scale. Political Geography, 1 febrero 1997, vol 16, no 2, pp. 92-97

Gough, J. (2004). Changing scale as changing class relations: variety and contradiction in the politics of scale. Political Geography, 1 febrero 2004, vol. 23, no 2, pp. 185-221

Harvey, D. (1985). The urbanization of capital: studies in the history and theory of capitalist urbanization, Baltimore. Maryland, Estados Unidos de América: Johns Hopkins University Press.

Harvey, D. (2005). The new imperialism. Oxford: Oxford University Press.

Keil, R. y Mahon, R. (2009). Leviathan Undone?: Towards a Political Economy of Scale. Vancouver, Canadá:

University of British Columbia Press.

Leitner, H. y Miller, B. (2007). Scale and the Limitation of Ontological Debate: A Commentary on Marston, Jones and Woodward. Transactions of the Institute of British Geographers, vol.

32, no. 1, pp. 116-125.

Marston, S. A., Jones III, J. P. y Woodward, K. (2005). Human geography without scale. Transactions of the Institute of British Geographers, 1 diciembre 2005, vol. 30, no. 4, pp.

Masson, D. (2009). Politique(s) des échelles et trasnationalisation: perspectives géographiques. Politique et Sociétés, vol. 28, no. 1, pp. 113-133.

Ripoll, F. (2005). De la planète finite aux espaces de vie: La dimension spatiale des militantismes pour la décroissance en France et au Québec. Caen, France: Université de Caen Basse-Normandie.

Smith, N. (1992). Contours of a Spatialized Politics: Homeless Vehicles and the Production of Geographical Scale. Social Texte, no 33, pp. 55-81

Smith, N. (1993). Homeless/global: Scaling Places. Mapping the futures: local cultures, global change, London, Routgledge, pp. 87-119.

Swyngedouw, E. (1997). Excluding the other: the production of scale and scaled politics. Geographies of economies. London: Arnold. Pp. 167-176

Swyngedouw, E. (2004). Globalisation or 'glocalisation'? Networks, territories and rescaling. Cambridge Review of International Affairs, vol. 17, no 1, p. 19

Taylor, P. J. (1982). A Materialist Framework for Political Geography. Transactions of the Institute of British Geographers, vol 7, no 1, pp. 15-34 


\section{Referencias de la edición en español}

González, S. (2005). La geografía escalar del capitalismo actual. Scripta Nova. Revista electrónica de geografía y ciencias sociales. Universidad de Barcelona, vol. IX, núm. 189. http://www. ub.edu/geocrit/sn/sn-189.htm

Laura Silveira, M. (2015). Escala geográfica: da ação ao império?. Terra Livre, 2(23), 87-96. Recuperado de https://publicacoes.agb.org.br/index.php/terralivre/article/view/195

Ruíz Rivera, N., \& Galicia Sarmiento, L. (2016). La escala geográfica como concepto integrador en la comprensión de problemas socio-ambientales. Investigaciones Geográficas, (89), 137. https://doi.org/10.14350/rig.47515

Santos, M. (1993). Los espacios de la globalización. Anales de Geografía de la Universidad Complutense, no. 13, 69-77. https://revistas.ucm.es/index.php/AGUC/issue/view/ AGUC939311

Smith, N. (2015). Geografia, diferencia y políticas de escala. Terra Livre, 2(19). Recuperado de https://publicacoes.agb.org.br/index.php/terralivre/article/view/162 